\title{
ENSACAMENTO DE FRUTOS DE ABIU VISANDO À PROTEÇÃO CONTRA O ATAQUE DA MOSCA-DAS-FRUTAS ${ }^{1}$
}

\author{
WALNICE MARIA OLIVEIRA DO NASCIMENTO², CARLOS HANS MÜLLER ${ }^{2}$, \\ CAROLINA DOS SANTOS ARAÚJO3, BRUNO CALZAVARA FLORES ${ }^{3}$
}

RESUMO- O abiu (Pouteria caimito) é fruto bastante conhecido nos trópicos e consumido in natura. Dentre os principais problemas que afetam a qualidade dos frutos, estão os danos causados por ataque da larva de mosca-das-frutas. O objetivo do trabalho foi determinar o diâmetro do fruto de abiu para efetivar a prática do ensacamento, visando à proteção contra o ataque da mosca. Para isto, foram estabelecidas seis classes de diâmetros: a) frutos com diâmetro menor que $1 \mathrm{~cm}$; b) frutos com diâmetro de $1 \mathrm{a} 2 \mathrm{~cm}$; c) frutos com diâmetro de 2 a $3 \mathrm{~cm}$; d) frutos com diâmetro de 3 a $4 \mathrm{~cm}$; e) frutos com diâmetro de 4 a $5 \mathrm{~cm}$; f) frutos com diâmetro maior que $5 \mathrm{~cm}$, e o tratamento-testemunha, que constou de frutos sem proteção. Os sacos para a cobertura dos frutos foram confeccionados em TNT, na cor branca. Após a colheita, os frutos foram medidos e pesados, e depois partidos nos sentidos longitudinal e transversal e avaliados por meio da observação visual, quanto à presença ou ausência de larva da mosca-das-frutas. A eficiência dos tratamentos foi avaliada pela porcentagem de frutos caídos ao chão, e de frutos colhidos com ausência e presença da larva. Foram observados 96,$7 ; 88,3$ e 40,0\% de queda nos frutos ensacados com diâmetro menor que $1 \mathrm{~cm} ; 1-2 \mathrm{~cm}$, e 2-3 $\mathrm{cm}$, respectivamente. $\mathrm{O}$ ensacamento de frutos de abiu com diâmetro entre $1 \mathrm{e} 2 \mathrm{~cm}$ não é viável devido à alta porcentagem de frutos caídos $(96,7 \%)$. A maior porcentagem de frutos colhidos sem larva da mosca-dasfrutas $(83,3 \%)$ é verificada quando os frutos foram ensacados com diâmetro entre três e cinco centímetros.

Termos para indexação: Anastrepha sp., cobertura, controle, praga, Pouteria caimito.

\section{FRUIT BAGGING OF Pouteria caimito AIMING PROTECTION AGAINST THE ATTACK OF FRUIT FLY}

\begin{abstract}
The Pouteria caimito tree, is a known fruit in the tropics and consumed fresh. Among the main issues affecting the fruit quality, are the damage caused by the attack of the larvae of fruit fly. The objective of this study was to determine the diameter of the fruit $P$. caimito to effect the practice of bagging in order to protect against the attack of the fly. For this, six classes of diameter were set: a) fruit with a diameter less than $1 \mathrm{~cm}, \mathrm{~b}$ ) fruit with a diameter of 1-2 cm, c) fruits with a diameter of 2-3 cm d) fruits with a diameter of 3 to $4 \mathrm{~cm}$, e) fruit with a diameter of $4-5 \mathrm{~cm}, \mathrm{f}$ ) fruit with a diameter larger than $5 \mathrm{~cm}$, and the control treatment, which consisted of fruit without protection. Bags to cover the fruits were made of white TNT. After harvest, fruits were measured and weighed and then broken in longitudinal and transversal directions and evaluated by visual observation of the presence or the absence of fruit fly larvae. The efficiency of treatments was evaluated by the percentage of fruit dropped on the ground, and the fruits harvested with absence and presence of larvae. It was observed $96.7 \%, 88.3 \%$ and $40.0 \%$ of dropping in bagged fruit with a diameter smaller than $1 \mathrm{~cm}, 1-2 \mathrm{~cm}$ and 2-3 cm respectively. The bagging of fruits $P$. caimito with diameters between 1 and $2 \mathrm{~cm}$ is not feasible due to the high percentage of fallen fruit $(96.7 \%)$. The highest percentage of fruits without larvae of fruit fly (83.3\%) is observed when the fruits were bagged with a diameter of between three and five centimeters.
\end{abstract}

Index terms: Anastrepha sp., covering, control, pest, Pouteria caimito.

\footnotetext{
${ }^{1}$ Trabalho (077-10). Recebido em: 23-03-2010. Aceito para publicação: 28-09-2010.

${ }^{2}$ Eng. Agro. Pesquisador da Embrapa Amazônia Oriental, Caixa Postal, 48, CEP 66095-100, Belém-PA. Emails: walnice@cpatu.embrapa.br; hans@cpatu.embrapa.br

${ }^{3}$ Bolsista da Embrapa Amazônia Oriental, Belém-PA. bionoma@hotmail.com; bruno.calzavara@hotmail.com
} 


\section{INTRODUÇÃO}

O abieiro, Pouteria caimito (RUIZ; PAVON) Radlk, pertence à família Sapotaceae. Seu provável centro de origem é a Amazônia Ocidental e zonas limítrofes do Peru, Colômbia e Venezuela (MANICA, 2000). Embora pouco explorada comercialmente, o abiu é uma fruta bastante consumida nos trópicos na forma in natura. No Brasil, seu consumo é mais difundido nos estados da região Norte, mais especificamente no Acre, Amapá e Pará.

Atualmente, existe no mercado apenas uma variedade disponível para cultivo: é a cultivar "graúdo" (DONADIO, 2000). Entretanto, a Embrapa Amazônia Oriental selecionou e vem desenvolvendo pesquisas com o clone Gigante-do-Solimões, que apresenta plantas com elevada produtividade, frutos grandes, com peso médio de $581,25 \mathrm{~g}$, pouco látex e rendimento percentual de parte comestível em torno de $60 \%$ (CARVALHO et al., 2010).

Dentre os principais problemas que afetam a qualidade dos frutos de abiu, destacam-se os danos causados por ataque de pragas. A mosca-das-frutas é considerada a praga de maior importância para a cultura do abieiro. Os danos diretos aos frutos são verificados internamente pelo apodrecimento da polpa e externamente pela perfuração e exsudação de látex coagulado, reduzindo o seu valor comercial e tornando-os impróprios para o consumo e comercialização, chegando a ocasionar $100 \%$ de perdas durante o período de safra.

Existem diversos gêneros de mosca que atacam as frutas, sendo o Anastrepha de maior importância no Brasil devido ao grande número de espécies e à ampla distribuição em todo o território nacional. As espécies que ocorrem na cultura do abieiro são Anastrepha fraterculus, Anastrepha leptozona, Anastrepha obliqua, Anastrepha striata e Anastrepha serpentina (GALLO, 2002; CARVALHO, 2005). O primeiro registro da incidência da mosca-das-frutas em frutos de abiu no Estado de S. Paulo foi feito por Raga et al. (2003), que na ocasião verificaram a ocorrência de duas espécies: A serpentina e A. leptozona.

$\mathrm{Na}$ literatura, são encontradas várias medidas para o controle da mosca-das-frutas, sendo o uso de produtos químicos, ainda, o principal método. Entretanto, embora este seja menos trabalhoso e oneroso ao produtor, os prejuízos causados ao meio ambiente e à saúde humana podem ser severos (CARVALHO et al., 2000). Como prática de controle da Anastrepha sp., Gallo et al. (2002) recomendam o ensacamento dos frutos, além de pulverização com inseticidas, suspendendo a aplicação 30 dias antes da colheita. Essa recomendação é feita genericamente para o controle de mosca-das-frutas em várias espécies de frutíferas. Entretanto, deve-se ressaltar que, no Ministério de Agricultura, Pecuária e Abastecimento (MAPA), ainda não há produto registrado para o controle dessa praga na cultura do abieiro.

Nos últimos anos, com a mudança no perfil do consumidor, particularmente os que consomem frutos in natura, tem aumentado a exigência por alimentos com níveis reduzidos ou mesmo isentos de agrotóxicos. Atualmente, o ensacamento dos frutos é alternativa mais eficaz para o manejo de mosca-dasfrutas. O que vem motivando e merecendo destaque é a realização de pesquisas com o aprimoramento da técnica do ensacamento de frutos para controle dessa praga em espécies frutíferas. Em diversas culturas, já existem resultados de pesquisas recomendando o ensacamento de frutos, principalmente para espécies frutíferas que apresentam frutos que podem ser consumidos in natura, como, por exemplo: goiaba, figo, carambola, pera e pêssego (PEREIRA; BORTOLI, 1998; MAZARO et al., 2005; DONADIO et al., 2001; COELHO et al., 2008).

$\mathrm{O}$ tipo de material mais apropriado para o ensacamento dos frutos também é objetivo de estudo por muitos pesquisadores. Coelho et al. (2008) trabalharam com sacos de TNT, polipropileno, polietileno e papel impermeável, e verificaram que todos os materiais foram eficientes como barreira mecânica ao ataque da mosca-das-frutas em frutos de pêssego. Malgarim e Mendes (2007) também obtiveram bons resultados quando utilizaram o TNT para o ensacamento de frutos de goiaba. Em caqui, o material que apresentou melhor resultado foi o saco de jornal. Além do tipo de material, tem sido dada ênfase ao tamanho adequado do fruto para o ensacamento, como o trabalho conduzido por Grassi (2008) com frutos de nêspera.

O objetivo deste trabalho foi determinar o diâmetro do fruto de $P$. caimito para a prática do ensacamento, visando à proteção contra o ataque da mosca-das-frutas.

\section{MATERIAL E MÉTODOS}

O experimento foi conduzido em nove plantas de abieiro do clone Gigante-do-Solimões, com sete anos de idade, estabelecidas na Embrapa Amazônia Oriental, no município de Belém-PA, no período de julho a agosto de 2009.

Foram estabelecidas seis classes de diâmetros para o ensacamento de frutos e o tratamentotestemunha, com a avaliação dos frutos colhidos maduros sem o ensacamento: a) frutos com diâmetro menor que $1 \mathrm{~cm}$; b) frutos com diâmetro de $1-2 \mathrm{~cm}$; 
c) frutos com diâmetro de $2-3 \mathrm{~cm}$; d) frutos com diâmetro de 3-4 cm; e) frutos com diâmetro de 4-5 cm; f) frutos com diâmetro maior que $5 \mathrm{~cm}$; g) testemunha (frutos sem ensacamento). $\mathrm{O}$ experimento constou de sete tratamentos. Os sacos para a cobertura dos frutos foram confeccionados com um tipo de tecido na cor branca, comercialmente denominado tecidonão-tecido, conhecido como TNT, com gramatura de $45 \mathrm{~g} / \mathrm{m}^{2}$.

Com o auxílio de um paquímetro digital, foi medido o diâmetro dos frutos e, em seguida, foram cobertos com os sacos de TNT, com dimensão de 20 $\mathrm{cm}$ de largura e $20 \mathrm{~cm}$ de comprimento, sendo fechados com grampos de metal na região do pedúnculo para evitar que as moscas tivessem acesso aos frutos.

À medida que atingiam a completa maturação, os frutos foram colhidos, mensurado o diâmetro com auxílio de paquímetro digital, pesados em balança de precisão de 0,1 e depois partidos nos sentidos longitudinal e transversal, e avaliados por meio da observação visual quanto à presença ou ausência de larva da mosca-das-frutas. Os dados relativos ao diâmetro e à massa dos frutos obtidos foram analisados através de estatística simples, envolvendo médias e desvio-padrão. A eficiência dos tratamentos foi avaliada pela porcentagem de frutos caídos ao chão antes de completar a maturação e de frutos colhidos maduros com presença e ausência da larva da mosca-das-frutas.

Adotou-se o delineamento experimental em blocos casualizado, com três repetições, sendo cada bloco constituído por três plantas, e cada parcela, por 20 frutos. Os dados foram expressos em porcentagem e transformados em arco-seno da raiz quadrada de $\mathrm{x}+\alpha / 100$. As médias foram submetidas à análise de variância e comparadas pelo teste de Tukey, a 5\% de probabilidade.

\section{RESULTADOS E DISCUSSÃO}

As médias obtidas para as mensurações feitas nos frutos antes do ensacamento e após a colheita estão disponibilizadas na Tabela 1 . Nos cinco tratamentos onde foi realizada a colheita, observase a produção de frutos com comprimento médio de $8,39 \mathrm{~cm}$, e peso de $345,92 \mathrm{~g}$. Entretanto, estes resultados ainda estão um pouco abaixo dos obtidos por Carvalho et al. (2010) com plantas do clone Gigante-do-Solimões.

Aos 51 dias após a aplicação dos tratamentos, foi realizada a colheita dos frutos maduros e verificouse que, quanto menor o diâmetro do fruto ensacado, maior foi a porcentagem de frutos caídos antes de completar a maturação (Figura 1). Nos frutos ensacados com diâmetro menor que $1 \mathrm{~cm}$, apenas $3,3 \%$ de frutos foram colhidos, $96,7 \%$ dos frutos caíram antes de completar a maturação. A grande quantidade de frutos caídos neste tratamento, provavelmente, ocorreu devido ao aborto seletivo da planta provocado pela limitação de recursos ou ao estresse fisiológico provocado pela cobertura dos frutos nos estádios iniciais de desenvolvimento (STEPHENSON; WINSON, 1986; CAVALCANTE et al., 2009).

No tratamento com frutos ensacados com tamanho maior que $5 \mathrm{~cm}$, foi encontrada a presença da larva em 53,3\% de frutos colhidos, sendo inferior apenas ao tratamento-testemunha (frutos sem proteção), onde foram registrados $98,3 \%$ dos frutos atacados pela mosca-das-frutas. É provável que a maior presença de larvas nos frutos ensacados com diâmetro acima de $5 \mathrm{~cm}$ seja atribuída à oviposição anterior à cobertura dos frutos, já que quanto mais precoce foi o ensacamento, menor foi o dano causado pela mosca-das-frutas, evidenciando a necessidade de se efetuar a proteção dos frutos de abiu.

Verificou-se que a maior proteção dos frutos contra o ataque da mosca-das-frutas ocorreu nos tratamentos quatro e cinco, com frutos ensacados com diâmetros de 3 a $4 \mathrm{~cm}$ e 4 a $5 \mathrm{~cm}$, com $83,3 \%$ e $76,7 \%$, respectivamente, de frutos colhidos sem larva. Entretanto, não houve diferença significativa entre os tratamentos (Tabela 2).

Diversos estudos foram feitos objetivando a determinação do tamanho adequado dos frutos para o ensacamento, visando à proteção ao ataque da mosca-das-frutas. Em experimento com nêspera, Grassi (2008) observou menor incidência da larva da mosca quando os frutos foram cobertos com diâmetros entre 1 a $2 \mathrm{~cm}$. Para frutos de abiu, os resultados indicam que o ensacamento deve ser feito quando os frutos apresentam diâmetro entre $3 \mathrm{a} 5 \mathrm{~cm}$, com 76,7 a 83,8\% de frutos colhidos sem a larva da mosca-das-frutas. Portanto, a maior proteção contra $o$ ataque da praga foi encontrada no tratamento em que a cobertura com sacos de TNT foi feita, quando os frutos apresentavam o diâmetro médio de $4,49 \mathrm{~cm}$, onde apenas $11,7 \%$ de frutos colhidos apresentavam larvas da mosca-das-frutas (Tabela 2).

A utilização dos sacos de TNT para a cobertura dos frutos de abiu possui como vantagens: o baixo custo e a simplicidade de confecção, além da reutilização dos mesmos em safras subsequentes. Recomendação semelhante foi feita por Malgarim e Mendes (2007), quando utilizaram sacos de TNT para a proteção de frutos de goiaba.

Os dados obtidos no experimento estão 
condizentes com os encontrados por Faoro (2003), em frutos ensacados de pera-japonesa, o qual observou redução nos danos provocados por fatores bióticos e abióticos, o que levou à obtenção de frutos da pera-japonesa de melhor qualidade interna e com aspecto visual mais agradável, além de reduzir a quantidade de agrotóxicos aplicados. Entretanto, cada produtor deverá considerar a relação custo/benefício para adotar a prática do ensacamento, observando os benefícios já citados.

TABELA 1 - Média e desvio-padrão obtidos para o diâmetro e peso em frutos de Pouteria caimito, por ocasião do ensacamento e na colheita. Belém-PA. ( $\mathrm{n}=60$ frutos).

\begin{tabular}{lccc}
\hline $\begin{array}{c}\text { Tratamento } \\
\text { (tamanho do fruto protegido) }\end{array}$ & $\begin{array}{c}\text { Diâmetro do fruto } \\
\text { no ensacamento } \\
(\mathrm{cm})\end{array}$ & $\begin{array}{c}\text { Diâmetro do fruto na } \\
\text { colheita } \\
(\mathrm{cm})\end{array}$ & $\begin{array}{c}\text { Peso do fruto na } \\
\text { colheita } \\
(\mathrm{g})\end{array}$ \\
\hline Menor que 1cm & $0,61( \pm 0,1)$ & $6,11( \pm 0,4)$ & $176,10( \pm 92,5)$ \\
1 a $2 \mathrm{~cm}$ & $1,28( \pm 0,2)$ & $6,99( \pm 0,4)$ & $192,40( \pm 37,1)$ \\
2 a $3 \mathrm{~cm}$ & $2,67( \pm 0,2)$ & $8,95( \pm 0,5)$ & $404,01( \pm 56,9)$ \\
3 a $4 \mathrm{~cm}$ & $3,43( \pm 0,3)$ & $8,86( \pm 1,0)$ & $382,24( \pm 117,2)$ \\
4 a $5 \mathrm{~cm}$ & $4,49( \pm 0,6)$ & $8,53( \pm 0,8)$ & $368,22( \pm 112,3)$ \\
Maior que 5 cm & $5,77( \pm 0,5)$ & $8,62( \pm 0,9)$ & $382,75( \pm 98,5)$ \\
\hline
\end{tabular}

TABELA 2 - Porcentagem de frutos de Pouteria caimito, caídos ao chão antes de completar a maturação e colhidos maduros com e sem a larva da mosca-das-frutas. Belém-PA.

\begin{tabular}{ccccc}
\hline $\begin{array}{c}\mathrm{N}^{\mathrm{O}} \\
\text { do }\end{array}$ & $\begin{array}{c}\text { Diâmetro } \\
\text { do fruto } \\
\text { Tratamento }\end{array}$ & $\begin{array}{c}\text { Fruto caído ao chão } \\
(\%)\end{array}$ & $\begin{array}{c}\text { Fruto colhido sem } \\
\text { larva }(\%)\end{array}$ & $\begin{array}{c}\text { Fruto colhido com } \\
\text { larva (\%) }\end{array}$ \\
\hline 1 & Menor que $1 \mathrm{~cm}$ & $96,7 \mathrm{a}^{*}$ & $3,3 \mathrm{c}$ & $0,0 \mathrm{c}$ \\
2 & $1 \mathrm{a} 2 \mathrm{~cm}$ & $88,3 \mathrm{ab}$ & $10,0 \mathrm{bc}$ & $1,7 \mathrm{c}$ \\
3 & $2 \mathrm{a} 3 \mathrm{~cm}$ & $40,0 \mathrm{abc}$ & $58,3 \mathrm{ab}$ & $1,7 \mathrm{c}$ \\
4 & $3 \mathrm{a} 4 \mathrm{~cm}$ & $11,7 \mathrm{bc}$ & $83,3 \mathrm{a}$ & $5,0 \mathrm{c}$ \\
5 & $4 \mathrm{a} 5 \mathrm{~cm}$ & $11,7 \mathrm{bc}$ & $76,7 \mathrm{a}$ & $11,6 \mathrm{c}$ \\
6 & Maior que $5 \mathrm{~cm}$ & $3,3 \mathrm{c}$ & $43,4 \mathrm{ab}$ & $53,3 \mathrm{~b}$ \\
Testemunha & Frutos sem proteção & $0,0 \mathrm{c}$ & $1,7 \mathrm{c}$ & $98,3 \mathrm{a}$ \\
\hline CV $(\%)$ & & 44,4 & 25,7 & 28,0 \\
\hline
\end{tabular}

* Médias seguidas das mesmas letra na coluna não diferem entre si, pelo teste de Tukey, a 5\% de probabilidade.

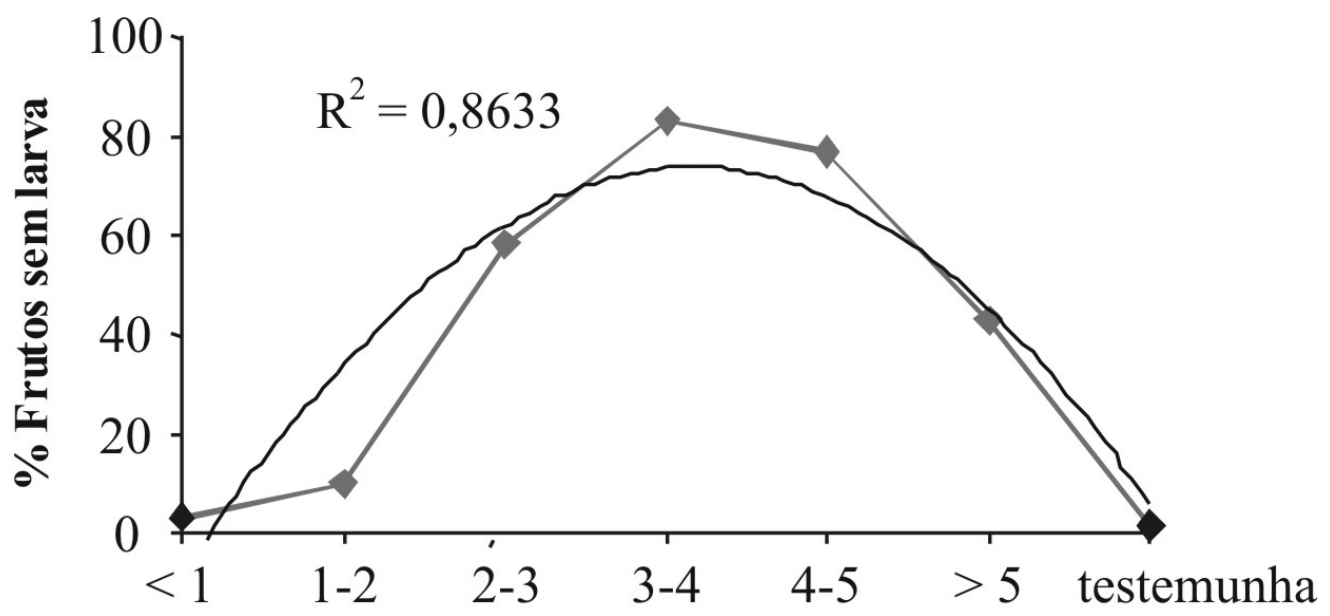

FIGURA 1 - Porcentagem de frutos de Pouteria caimito, colhidos sem larva da mosca-das-frutas. Belém-PA. 


\section{CONCLUSÕES}

1-O ensacamento de frutos de Pouteria caimito com diâmetro inferior a três centímetros não é viável devido à grande porcentagem de frutos caídos.

2-O ensacamento do fruto não elimina completamente o dano ocasionado pela Anastrepha sp. em frutos do abieiro.

3-Para melhor proteção contra o ataque da mosca-das-frutas, o ensacamento deve ser feito quando os frutos apresentam diâmetro entre três e cinco centímetros.

\section{AGRADECIMENTOS}

Os autores agradecem aos estudantes de nível médio do curso Técnico Florestal da Escola Juscelino Kubischek de Oliveira, Maria da Providência Lopes de Brito e Wasceny Lima Correia, pelo ensacamento dos frutos e coleta de dados em campo.

\section{REFERÊNCIAS}

CARVALHO, J. E. U.; NASCIMENTO, W. M. O. do; MÜLLER, C. H. Abieiro. Jaboticabal: Funep/ SBF, 2010. 33p. (Série Frutas Nativas, 5).

CARVALHO, R. S.; NASCIMENTO, A. S.; MATRANGOLO, W. J. R. Controle Biológico. In: MALAVASI, A. ZUCCHI, R. A. Moscas-das-frutas de importância econômica no Brasil: Conhecimento básico e aplicado. Ribeirão Preto: Holos, 2000. p.113-117.

CAVALCANTE, T. R. M.; V; NAVES, R. V.; FRANCESCHINELLI, E. V.; SILVA, R. P. da. Polinização e formação de frutos em araticum. Bragantia. Campinas, v. 68, n.1, p.13-21, 2009.

COELHO, L. R.; LEONEL, S.; CROCOMO, W. B.; LABINAS, A. M. Controle de pragas do pessegueiro através do ensacamento dos frutos. Ciência Agrotécnica, Lavras, v. 32, n. 6, p. 1743-1747, 2008.

DONADIO, L. C. Abiu graúdo. In: DONADIO, L. C. Novas variedades de frutas. Jaboticabal: SBF, 2000. p.18-21.
DONADIO, L. C.; SILVA, J. A. A.; ARAÚJO, P. S. R.; PRADO, R. M. Caramboleira (Averrhoa carambola L.). Jaboticabal: SBF, 2001. 81p.

FAORO, I. D. Técnica e custo para o ensacamento de frutos de pera-japonesa. Revista Brasileira de Fruticultura, Jaboticabal, v. 25, n. 2, p.339-340, 2003.

GALLO, D.; NAKANO, O.; SILVEIRA NETO, S.; CARVALHO, R. P. L.; BAPTISTA, G. C.; BERTI FILHO, E.; PARRA, J. R. P.; ZUCCHI, R. A.; ALVES, S. B.; VENDRAMIM, J. D.; MARCHINI, L. C.; LOPES, J. R. S.; OMOTO, C. Entomologia agrícola. Piracicaba: FEALQ, 2002. 920p.

GRASSI, A. M. Fenologia e qualidade de frutos de nespereira. 2008. 72 f. Dissertação (Mestrado em Fitotecnia) - Escola Superior de Agricultura Luiz de Queiroz, Universidade de São Paulo, Piracicaba, 2008.

MALGARIM, B.; MENDES, C. D. Ensacamento de goiabas visando ao manejo ecológico das moscasdas-frutas. Revista Brasileira de Agroecologia. Porto Alegre, v.2, n.2, p.706-709, 2007.

MANICA, I. Frutas nativas, silvestres e exóticas 1. Porto Alegre: Cinco Continentes Editora, 2000. $327 \mathrm{p}$.

MAZARO, S. M.; GOUVÊA, A.; CITADIN, I.; DANNER, M. A. Ensacamento de figos c.v. roxo de Valinhos. Scientia Agraria, Curitiba, v.6. n.1/2, p.59-63, 2005.

PEREIRA, F. M.; BORTOLI, S. A. Pragas da goiabeira. In: BARGA SOBRINHO, R.; CARDOSO, J. E.; FREIRE, F. C. O. (Ed.). Pragas de fruteiras tropicais de importância agroindustrial. Brasília: Embrapa-SPI, 1998. p.119-130.

RAGA, A.; MACHADO, R. A.; COSTA, A. A.; SOUZA FILHO, M. F.; VEIGA, R. F. A.; SAES, L. A. Primeiro relato de ocorrência de Anastrepha serpentina e Anastrepha leptozona (Dip.: Tephritidae) em abiu (Pouteria caimito) no Estado de São Paulo. Revista Brasileira de Fruticultura. Jaboticabal, v. 25, n.2, p.337-338, 2003.

STEPHENSON, A. G.; WINSON, J. A. Lotus corniculatus regulates offspring qualitythrough selective fruit abortion. Evolution, Amsterdam, v.40, p.453-458, 1986. 